\title{
Correction to: Predicting protein inter- residue contacts using composite likelihood maximization and deep learning
}

Haicang Zhang ${ }^{1,2}$, Qi Zhang ${ }^{1,2}$, Fusong Ju ${ }^{1,2}$, Jianwei Zhu ${ }^{1,2}$, Yujuan Gao ${ }^{3}$, Ziwei Xie ${ }^{5}$, Minghua Deng ${ }^{3}$, Shiwei Sun ${ }^{1 *}$, Wei-Mou Zheng ${ }^{4^{*}}$ and Dongbo $\mathrm{Bu}^{1,2^{*}}$

\section{Correction to: BMC Bioinformatics (2019) 20:537 https://doi.org/10.1186/s12859-019-3051-7}

Following publication of the original article [1], the author explained that there are several errors in the original article;

1. The figures' order in HTML and PDF does not match with each other.

2. The figures are incorrect order; the images do not match with the captions.

In this correction article the figures are shown correct with the correct captions.

\author{
Author details \\ 'Key Lab of Intelligent Information Processing, Institute of Computing \\ Technology, Chinese Academy of Sciences, Beijing, China. ${ }^{2}$ University of \\ Chinese Academy of Sciences, Beijing, China. ${ }^{3}$ Center for Quantitative \\ Biology,School of Mathematical Sciences, Center for Statistical Sciences, \\ Peking University, Beijing, China. ${ }^{4}$ Institute of Theoretical Physics, Chinese \\ Academy of Sciences, Beijing, China. ${ }^{5}$ College of Life Science and \\ Technology, Huazhong University of Science and Technology, Wuhan, China.
}

Published online: 29 November 2019

\section{Reference}

1. Zhang $\mathrm{H}$, et al. Predicting protein inter-residue contacts using composite

likelihood maximization and deep learning. BMC Bioinformatics. 2019;20:537. https://doi.org/10.1186/s12859-019-3051-7.

The original article can be found online at https://doi.org/10.1186/s12859019-3051-7

* Correspondence: dwsun@ict.ac.cn; zheng@itp.ac.cn; dbu@ict.ac.cn

${ }^{1}$ Key Lab of Intelligent Information Processing, Institute of Computing Technology, Chinese Academy of Sciences, Beijing, China

${ }^{4}$ Institute of Theoretical Physics, Chinese Academy of Sciences, Beijing, China

Full list of author information is available at the end of the article

(c) The Author(s). 2019 Open Access This article is distributed under the terms of the Creative Commons Attribution 4.0 International License (http://creativecommons.org/licenses/by/4.0/), which permits unrestricted use, distribution, and reproduction in any medium, provided you give appropriate credit to the original author(s) and the source, provide a link to the Creative Commons license, and indicate if changes were made. The Creative Commons Public Domain Dedication waiver (http://creativecommons.org/publicdomain/zero/1.0/) applies to the data made available in this article, unless otherwise stated. 

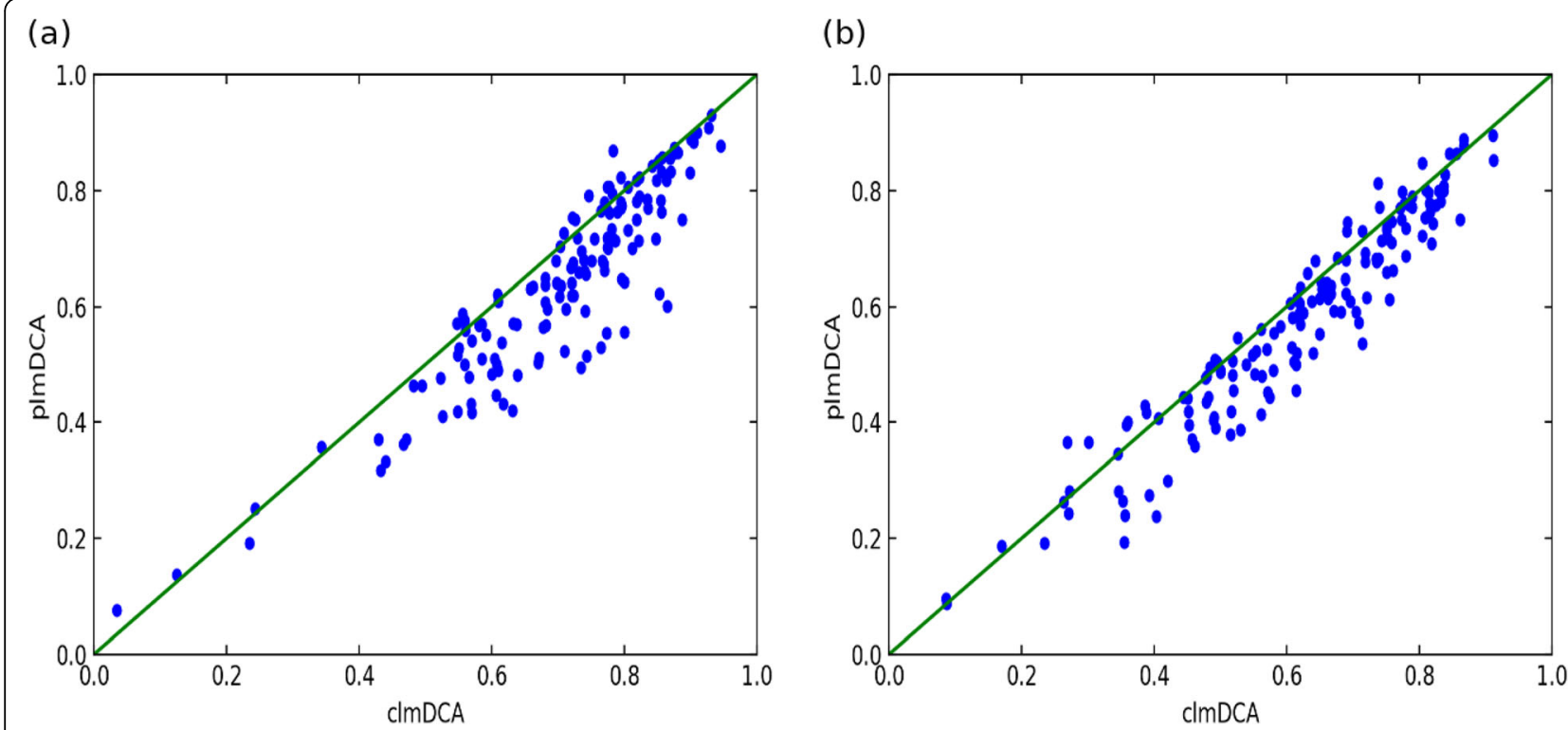

Fig. 1 Comparison of prediction accuracy of top L/2 contacts reported by plmDCA(y-axis) and $\operatorname{ImDCA}(x$-axis) with two sequence separation threshold on the PSICOV dataset. a Sequence separation $>6$ AA. b Sequence separation $>23$ AA

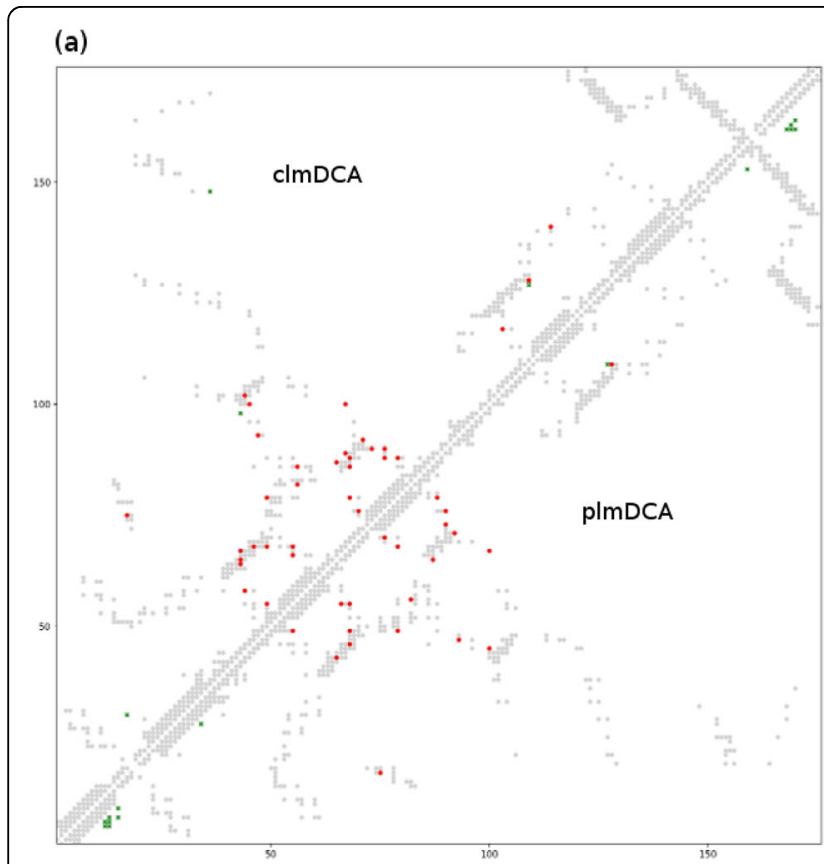

(b)

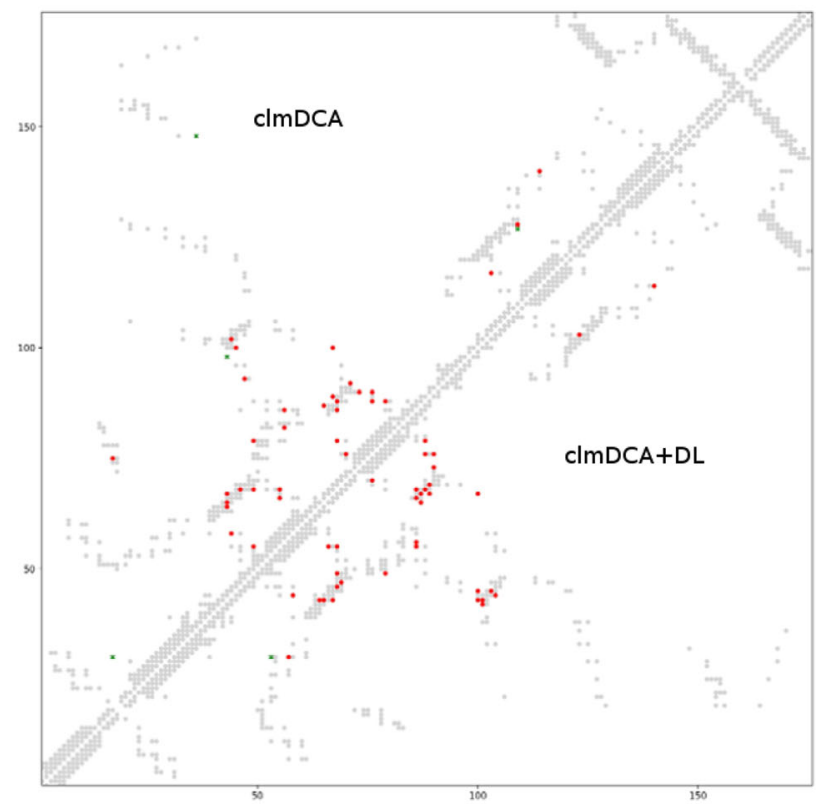

Fig. 2 Predicted contacts (top L/5; sequence separation $>6$ AA) for protein structure with PDB ID: 1ne2A by plmDCA and clmDCA. Red (green) dots indicate correct (incorrect) prediction, while grey dots indicate all true residue-residue contacts. a The comparison between clmDCA (in upper-left triangle) and plmDCA (in lower-right triangle). b The comparison between clmDCA (in upper-left triangle) and clmDCA after refining using deep residual network (in lower-right triangle) 

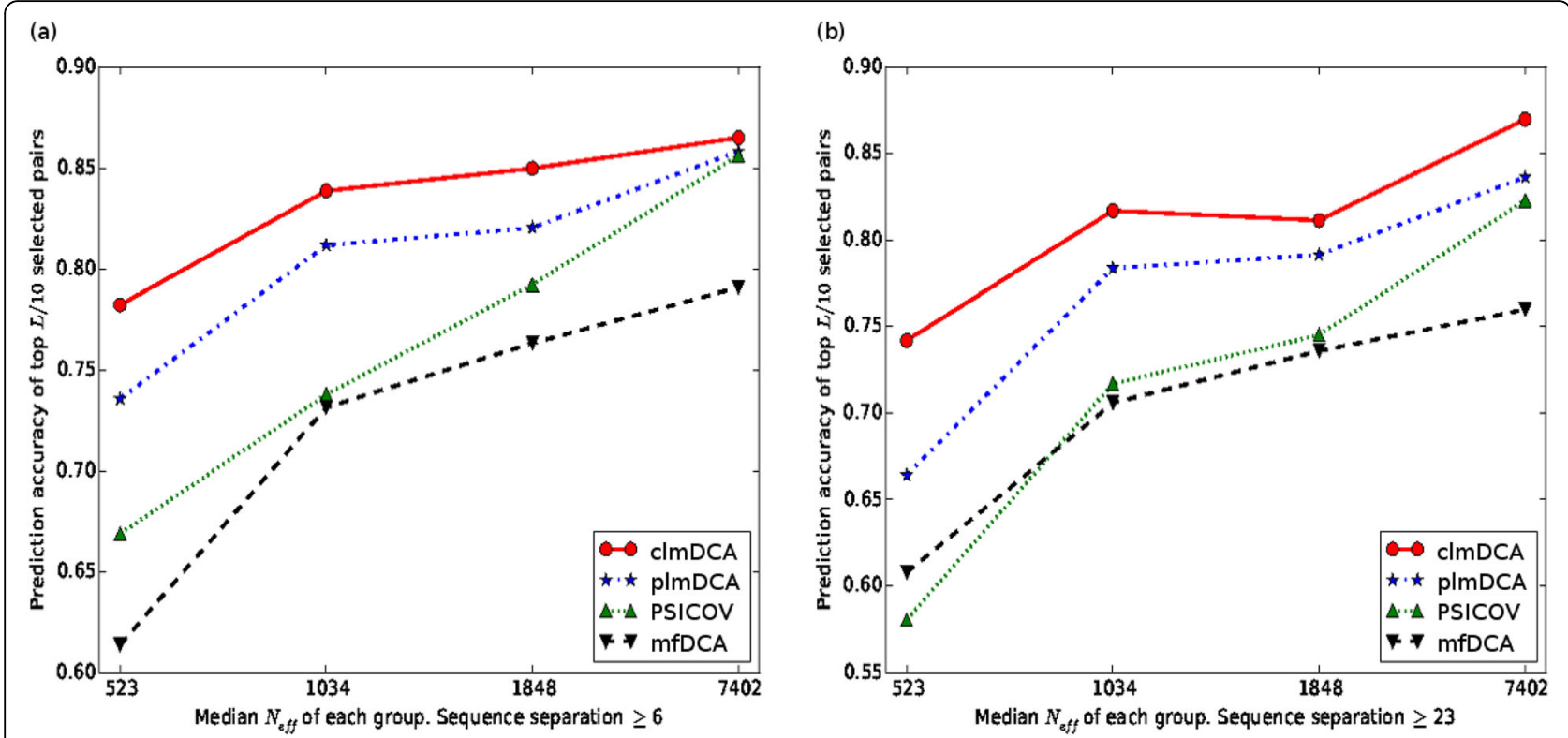

Fig. 3 The relationship between the prediction accuracy and quality of MSA. Here the quality of MSA is measured using Neff, i.e. the number of effective homologous sequences. Dataset: PSICOV. Sequence separation: > 6 AA 


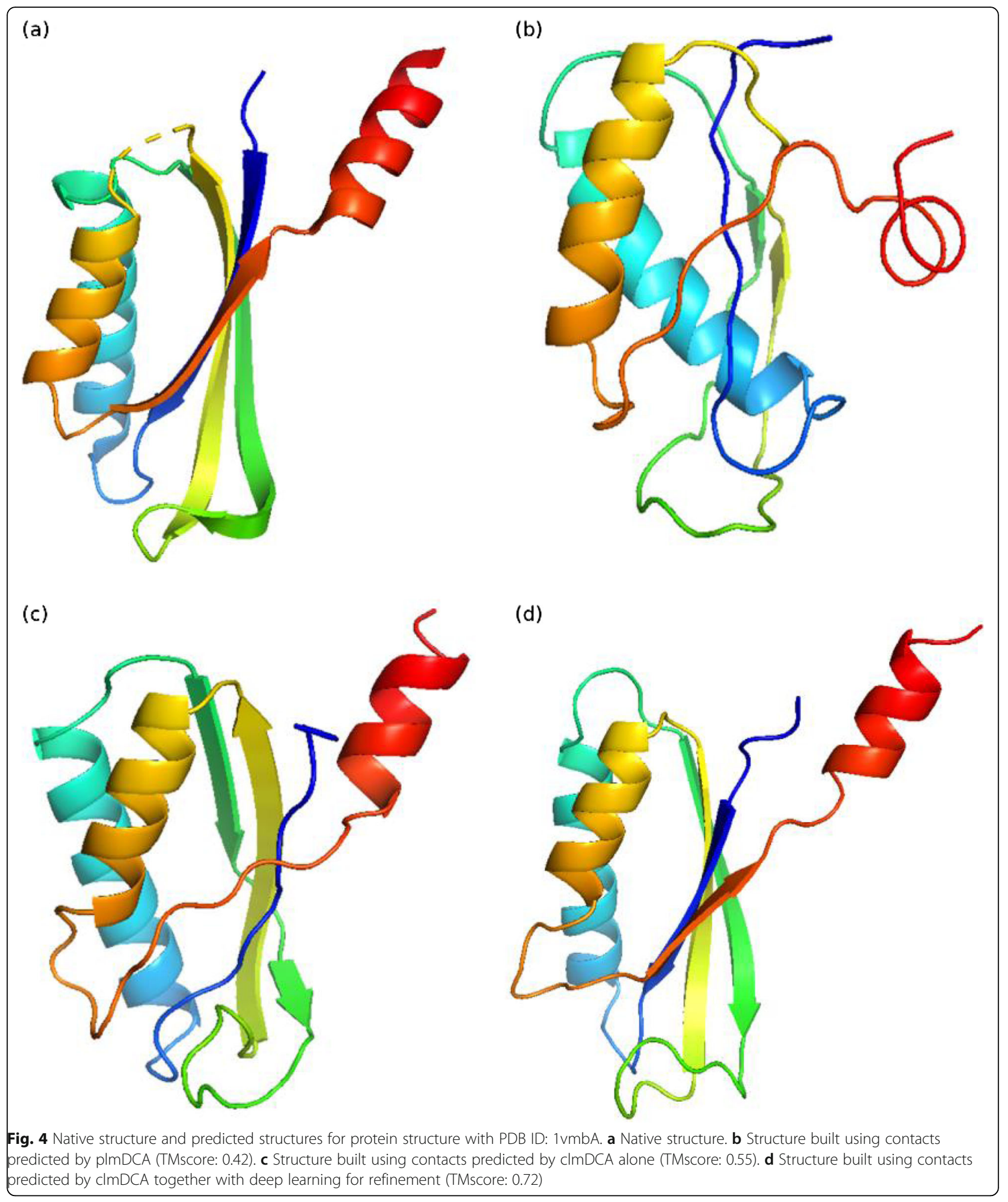




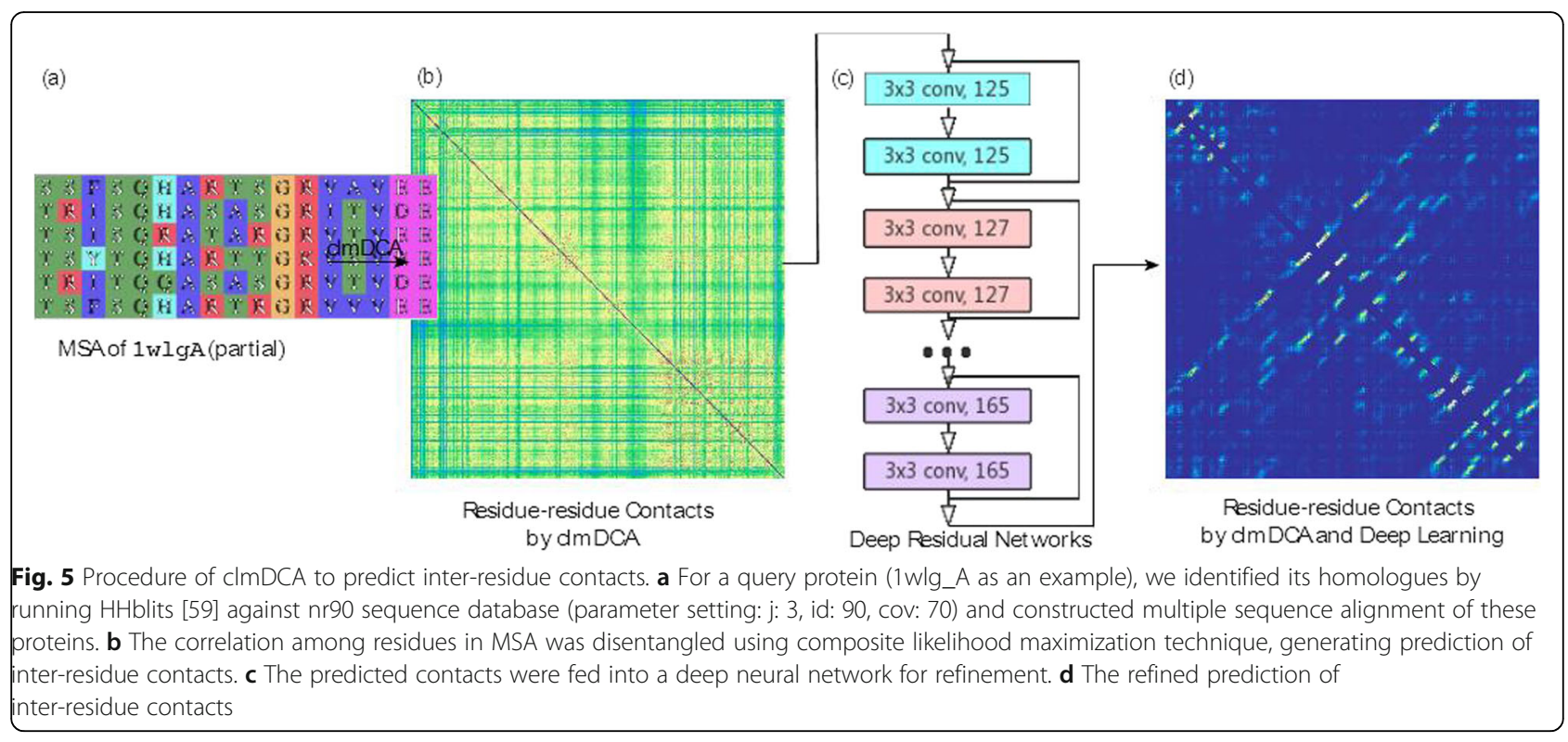

